\title{
植物遗传多样性与生态系统功能关系的研究进展
}

\author{
张俪文 韩广轩* \\ 中国科学院烟台海岸带研究所, 中国科学院海岸带环境过程与生态修复重点实验室, 山东烟台 264003
}

摘 要 全球变化和人类活动导致物种生境的萎缩, 造成很多植物种群数量缩减, 遗传多样性快速丧失。对于物种多样性低 的生态系统, 优势种的遗传多样性可能比物种多样性对生态系统功能产生更大的影响。因此, 了解遗传多样性和生态系统功 能的关系(GD-EF)及其机制对生物多样性保护、应对环境变化和生态修复具有指导意义。该文综述了植物遗传多样性对生态 系统结构(高营养级生物群落结构)和生态系统功能(初级生产力、养分循环和稳定性)的影响及机制、功能多样性对GD-EF的 影响、遗传多样性效应和物种多样性效应的比较, 以及GD-EF在生态修复等实际应用的研究进展。最后指出当前研究的不足 之处, 以期为后续研究提供参考：1)还需深入研究GD-EF机制; 2)未评估遗传多样性对生态系统多功能性的影响；3)不同遗传 多样性测度对生态系统功能的影响不明确; 4)缺少长期的和多空间尺度结合的GD-EF实验; 5)遗传多样性效应相对于其他因 子的作用不清楚。

关键词＼cjkstart遗传多样性；基因型多样性；群落结构；生态系统功能；加性效应；互补效应

张俪文, 韩广轩 (2018). 植物遗传多样性与生态系统功能关系的研究进展. 植物生态学报, 42, 977-989. DOI: 10.17521/cjpe.2018.0013

\section{A review on the relationships between plant genetic diversity and ecosystem functioning}

\author{
ZHANG Li-Wen and HAN Guang-Xuan* \\ Key Laboratory of Coastal Environmental Processes and Ecological Remediation, Chinese Academy of Sciences, Yantai Institute of Coastal Zone Research, \\ Chinese Academy of Sciences, Yantai, Shandong 264003, China
}

\begin{abstract}
The loss of genetic diversity is accelerating due to habitat loss and population reduction caused by global change and anthropologenic activities. For species-poor ecosystems, the effect of genetic diversity on ecosystem functioning may not be smaller than that of species diversity. Therefore, understanding the relationship between genetic diversity and ecosystem functioning (GD-EF) and its underlying mechanisms is important for biodiversity conservation, responses of ecosystems to environmental change and ecological restoration. Here, we reviewed the studies on the effects of plant genetic diversity on ecosystem structures (community structure of the higher tropic level) and ecosystem functions (primary production, nutrient cycling and ecosystem stability), and the mechanisms underlying these relationships. We also discussed the influence of functional diversity on GD-EF, the comparison of effects of the genetic and species diversity on ecosystem functioning, and the application of GD-EF in the ecological restorations. We finally pointed out the limitations in current studies to provide references for the future: (1) further studies on the mechanisms of GD-EF are needed; (2) no study has evaluated the influence of genetic diversity on maltifunctinarity; (3) the impacts of different measurements of genetic diversity on ecosystem functioning are unclear; (4) there are lack of long-time GD-EF studies and GD-EF studies conducted at multidimensional scales; (5) the relative importance of genetic diversity and other factors on ecosystem functioning in the nature is unclear.
\end{abstract}

Key words genetic diversity; genotypic diversity; ecosystem structure; ecosystem function; additive effect; complementarity effect 
生物多样性包括了三个层面: 遗传多样性、物 种多样性和生态系统多样性。由于人口增长、气候 变化以及掠夺式的土地利用导致生境减少乃至丧失, 使得生物种群个体数迅速减少、甚至物种快速灭绝, 生物多样性在三个层面上同时空前丧失。生物多样 性的减少对生态系统功能造成什么样的影响成为备 受关注的问题(Butchart et al., 2010; Hughes \& Stachowicz, 2011; Tilman et al., 2014)。因此, 生物多样 性与生态系统功能的关系是生态学研究的一个热点, 了解和掌握生物多样性与生态系统功能的关系及其 内在机制对于生物多样性、生态系统保护及其合理 利用意义重大。纵观生物多样性与生态系统功能关 系的研究, 大多集中在物种多样性与生态系统功能 关系上。国内学术期刊关于物种多样性与生态系统 功能关系的综述将近20篇, 从多个角度阐述了物种 多样性和生态系统功能关系、机制及其研究进展。 虽然物种多样性也是遗传多样性的一种测度, 但是 不能代表种群内的遗传多样性, 因为遗传多样性代 表的是物种进化的可能性。而且也不能简单地将物 种多样性与生态系统功能关系的研究结果套用在遗 传多样性与生态系统功能的关系(GD-EF)及其内在 机制上, 尽管目前有关GD-EF的实验方法和机制假 说多借鉴物种多样性与生态系统功能关系的研究。 探讨GD-EF及其内在机制能够完善生物多样性与生 态系统功能的关系的研究, 增加人类对自然生态系 统规律的认知, 而且植物优势种的遗传多样性对于 环境恶劣、物种数少、群落组成单一的生态系统(如: 农田、滨海湿地、海洋、沙漠、高寒山地和干早地 区)功能的影响可能与物种多样性的作用同等或更 重要。然而, 相对物种多样性与生态系统功能关系 的研究, 有关GD-EF及其内在机制的探讨较少 (Hughes \& Stachowicz, 2004; 沈栋伟等, 2007)。国内 有关综述只有沈栋伟等(2007)介绍了植物种群克隆 多样性及其影响因素、植物克隆多样性对生态系统 功能关系的影响及其机制。本文就近十几年GD-EF 实验研究进展进行综述。

\section{1 植物遗传多样性与生态系统功能关系及 其机制}

多年来群落遗传学领域的学者们致力于将微观 的基因和宏观的群落乃至生态系统关联起来, 试图 结合进化生物学和生态学来揭示群落和生态系统的
格局及机制(Whitham et al., 2006; Bailey et al., 2009; Bailey, 2011; Hersch-Green et al., 2011)。遗传多样性 研究不再只关注遗传变异在种群进化中的作用, 即 遗传变异如何影响种群个体的功能性状从而影响种 群适合度, 同时群落生态学、生态系统生态学的研 究也不再满足只探究物种水平、群落水平、生态系 统水平上的格局和机制, 希望更深入地从个体、基 因层面去探索和解决一些宏观生态学的科学问题。 其中, 探索GD-EF是一个很好的切入点。

早在 1859 年, 达尔文指出混种几个小麦 (Triticum aestivum)品种的土地比单一小麦品种的土 地产量更高(Darwin, 1859)。20世纪八九十年代, 农 学家们通过在一块土地上混合种植一种农作物的多 个品种来减少病虫害增加粮食产量(Wolfe, 1985; Smithson \& Lenné, 1996)。无独有偶, 保护生物学家 也在试图通过增加稀有物种种群的遗传多样性来提 高稀有物种种群的适合度(Lande, 1988)。但是学者 们对GD-EF的浓厚兴趣还得追溯到1990年后, 分子 遗传标记技术(包括中性和适应性分子标记技术)逐 渐发展成熟，提高了对遗传分析和基因型的分辨、 检测能力, 非常有利于GD-EF实验开展和研究(沈 栋伟等, 2007)。经过十多年的探索, GD-EF实验研究 取得了许多进展。

\section{1 遗传多样性对生态系统结构的影响及机制}

植物遗传多样性丧失对高营养级生物群落结构 (包括物种多样性和物种组成等)影响如何是大家非 常关注的问题。大多数研究表明, 植物基因型丰富 度(相关名词解释请见表1)的提高能够增加高营养 级动物(比如节肢动物)的丰富度和多度, 从而改变 生态系统结构, 其内在机制可能为非加性效应或者 加性效应和非加性效应皆有(Loreau \& Hector, 2001; Crutsinger et al., 2006; Johnson et al., 2006; Crawford \& Rudgers, 2013; Wang et al., 2014; Hughes et al., 2016; 申俊芳等, 2016)。

加性效应和非加性效应是从生物学角度进行的 分类(表2)。加性效应是指不同基因型组成的生态系 统功能效应不一样, 但它们的效应是独立的, 即不 同基因型间的相互作用对生态系统功能没有影响, 因而它们的生态系统效应是可加的。优势效应(Fox, 2005), 也称为选择效应(Huston, 1997)属于加性效 应, 其指基因型多样性高的系统中包含有利基因型 
表1 植物遗传多样性与生态系统功能关系的相关术语解释

Table 1 The explanation of the plant genetic diversity-ecological functioning glossary

\begin{tabular}{ll}
\hline 名词术语 Glossary & 解释 Explanation \\
\hline 遗传多样性 Genetic diversity & 种群内个体间遗传变异的程度。 \\
& The degree of genetic variation among individuals in a population. \\
& 所检测位点上等位基因的平均数目。 \\
& The average number of alleles detected at the detected locus.
\end{tabular}

基因多样性 Allelic diversity

基因型 Genotype

基因型丰富度 Genotypic richness

基因型均匀度 Genotypic eveness

基因型相异度 Genotypic dissimilarity

基因型亲缘度 Genotypic relatedness

适应性遗传多样性

Adaptive genetic diversity

中性遗传多样性 Neutral genetic diversity

品种 Cultivar

近交衰退 Inbreeding depression

远交衰退 Outbreeding depression

功能多样性 Functional diversity

奠基者多样性 Founder diversity

系统发育多样性 Phylogenetic diversity
包含位点上基因数目和频率信息的一类遗传多样性指数, 比如: Shannon信息指数和期望杂合度。 A class of genetic diversity indices containing information about the number and frequency of genes at a locus, such as: Shannon information index and expected heterozygosity.

一个个体在指定数量的位点上等位基因的组成。

The composition of alleles of an individual at a specified number of loci.

一个种群中基因型的数目。

The number of genotypes in a population.

基因型多度的分布。如果一个种群中各基因型多度等同, 那么基因型均匀度为 1 ; 如果一个种群只有一个基 因型，那么该种群基因型均匀度为 0 。

The distribution of genotypic abundance. The genotype evenness is 1 if genotypic abundance is equal in a population, and 0 if there is only one genotype in a population.

一个种群中两两基因型间遗传距离的平均值。

The average genetic distance between two genotypes in a population.

与基因型相异度相反, 指一个种群中两两基因型间亲缘程度的平均值。对于植物微卫星分子标记数据, 二 倍体可以用STORM软件(Frasier, 2008)或者R程序包“related” (Pew et al., 2015), 多倍体用P $P_{\text {OLY R }}$ ELATEDNESs 计 算基因型亲缘度(Huang et al., 2015)。

The average value of relatedness between two genotypes in a population, which is contrary to genotypic dissimilarity. For plant microsatellite marker data, genotypic relatedness of diploids can be calculated with STORM software (Frasier, 2008) or R package "related" (Pew et al., 2015), and genotypic relatedness of polyploids can be calculated with POLYR RLATEDNESS (Huang et al., 2015).

适应性遗传多样性是通过改变表达蛋白质的数量或结构或表达时间来影响表型以帮助个体适应环境或者 提高个体适合度的变异。一般采用已知遗传关系的个体(比如, 来自同一母株种子生长的个体)开展同质园 数量遗传实验进行估算, 但是这个方法比较费时费力费钱; 另外一种方法是开发和篮选出适应性分子标记 来测定其遗传变异。

Adaptive genetic diversity is the variation that affects phenotypes by altering the number or structure of expressed proteins or the expression time to help individuals adapt to the environment or improve their fitness. Quantitative genetic common garden experiments are usually conducted to estimate the adaptive gentic variation by using individuals with known genetic relationships (e.g., individuals from seeds of the same mother tree), but this method is time-consuming and costly; another method is to develop and select adaptive molecular markers to determine their genetic variation.

中性遗传多样性是由不影响表型的序列变异组成。中性遗传多样性和适应性遗传多样性也可能有相关性, 原因是采用的中性分子标记位点与适应性遗传变异可能存在连锁不平衡的情况。

Neutral genetic diversity is composed of sequence variations that do not affect phenotypes. Neutral genetic diversity and adaptive genetic diversity may also be correlated, because there may be linkage imbalance between the neutral molecular marker loci and adaptive genetic variation.

为特定的某一性状或若干性状的组合而选择出来的植物集合体, 在这些性状上是特异、一致、稳定的, 并 且通过适当的有性或无性方式繁殖时仍保持这些性状。

Plants were selected for a particular trait or combination of several specific traits, and these traits are specific, consistent, and stable, and retained when propagated sexually or asexually.

由于近交(自交和亲缘个体间的异交)导致后代适合度下降的现象, 产生的主要原因是由于近交增加了有害 等位基因的纯合几率。

Inbreeding (selfing and outcrossing between related individuals) results in a decrease in fitness of offspring, mainly because inbreeding increases the probability of harmful homozygous alleles.

不同生境的种群个体, 各自拥有适应当地生境的特有等位基因组合, 如果它们相互之间杂交(交配)将可能 打破这种组合, 引起后代适应能力降低。

Individuals from different habitats have specific allele combinations adapted to local habitats. If they cross breeding (mate) with each other, they may break the specific allele combinations and reduce the adaptability of their offspring.

植物个体水平上的形态、生理以及生活史特征等功能性状通过影响植物存活能力、生长和繁殖来影响其适 合度, 这些功能性状特征值的大小、范围和分布状况称为功能多样性。

Functional traits such as morphology, physiology and life history at the individual level affect plant fitness by affecting its survival, growth and reproduction. The size, range and distribution of these functional trait values are called functional diversity.

这里指的是, 在种群保护或者生态修复中, 所引物种种群的遗传多样性。奠基者效应是指由少数个体的基 因频率决定了它们后代基因频率的效应。

This refers to the genetic diversity of founder in population conservation or ecological restoration. Founder effect is the gene frequency of a small number of individuals determines the gene frequency of their offspring.

群落中物种的系统发育树形图(表示物种之间的亲缘关系)中所有分枝长度之和。

The sum of all the branch lengths in a phylogenetic tree of species in a community (representing the relatedness between species). 
表2 主要遗传多样性-生态系统功能关系机制假说

Table 2 Main genetic diversity-ecosystem functioning hypotheses

\begin{tabular}{|c|c|}
\hline $\begin{array}{l}\text { 效应类别(按生物 } \\
\text { 学角度来分类) } \\
\text { Types of genetic } \\
\text { diversity effects (in } \\
\text { terms of biology) }\end{array}$ & $\begin{array}{l}\text { 效应区别 } \\
\text { The differences among types of } \\
\text { genetic diversity effect }\end{array}$ \\
\hline $\begin{array}{l}\text { 加性效应 } \\
\text { Additive effects }\end{array}$ & $\begin{array}{l}\text { 不同基因型对生态系统功能的 } \\
\text { 效应是独立的、可加的; 基因型 } \\
\text { 间相互作用不影响其生态系统 } \\
\text { 功能效应。 } \\
\text { The effects of different genotypes } \\
\text { on ecosystem functioning are in- } \\
\text { dependent and additive, and the } \\
\text { interactions among genotypes do } \\
\text { not affect their effects on ecosys- } \\
\text { tem functioning. }\end{array}$ \\
\hline 非加性效应 & 不同基因型对生态系统功能效 \\
\hline $\begin{array}{l}\text { Non-additive } \\
\text { effects }\end{array}$ & $\begin{array}{l}\text { 应是非独立的、不可加的; 基因 } \\
\text { 型间相互作用对生态系统功能 } \\
\text { 产生交互效应。The effects of } \\
\text { different genotypes on ecosystem } \\
\text { functioning are dependent and } \\
\text { nonadditive, and the interactions } \\
\text { among genotypes affect their ef- } \\
\text { fects on ecosystem functioning. }\end{array}$ \\
\hline
\end{tabular}

\begin{tabular}{|c|c|c|}
\hline $\begin{array}{l}\text { 生态系统功能 } \\
\text { Ecosystem } \\
\text { functioning }\end{array}$ & $\begin{array}{l}\text { 相关机制 } \\
\text { 假说 } \\
\text { Hypothesis }\end{array}$ & $\begin{array}{l}\text { 假说内容 } \\
\text { Content of hypothesis }\end{array}$ \\
\hline $\begin{array}{l}\text { 高营养级生物群落 } \\
\text { 结构、初级生产力、 } \\
\text { 养分循环、生态系统 } \\
\text { 稳定性 } \\
\text { Community structure } \\
\text { of higher trophic } \\
\text { levels, primary pro- } \\
\text { ductivity, nutrientcy- } \\
\text { cling and ecosystem }\end{array}$ & $\begin{array}{l}\text { 优势效应 } \\
\text { Dominant } \\
\text { effect }\end{array}$ & $\begin{array}{l}\text { 基因型多样性高的系统中包含对系统有利的基因型概 } \\
\text { 率高于单基因型系统, 因此基因型多样性高有利于维 } \\
\text { 持生态系统结构和功能。验证方法请见1.1.和1.2节。 } \\
\text { Systems with high genotypic diversity have a higher } \\
\text { probability of containing genotypes beneficial to the } \\
\text { system than those with single genotype, and thus high } \\
\text { genotypic diversity is conducive to maintaining ecosys- } \\
\text { tem structure and function. Please see sections } \mathbf{1 . 1} \text { and } \\
\mathbf{1 . 2} \text { for the methods for testing this hypotheses. }\end{array}$ \\
\hline
\end{tabular}
stability

高营养级生物群落 结构

Community structure of higher trophic levels

初级生产力、养分循 环、生态系统稳定性 Primary productivity, nutrient cycling and ecosystem stability
资源化假说

The resource specialization hypothesis

大多数植食性节肢动物表现出一定程度的食性偏好, 随着基因型多样性的增加, 相关联的植食性节肢动物 多样性也增加。验证方法请见1.1节。

Most of the herbivorous arthropods showed food preference. With the increase of genotypic diversity, the diversity of associated herbivorous arthropods also increased. Please see Section $\mathbf{1 . 1}$ for the methods for testing this hypothesis.

更多个体假说 如果地上净生产力随着基因型多样性升高, 那么能够 The more 提供更多的能量给更多植食性节肢动物, 这些植食性 individuals 节肢动物多样性随之增加, 继而捕食者也会增加。验 hypothesis 证方法请见1.1节。

If net aboveground productivity increases with genotypic diversity, more energy can be provided to more herbivorous arthropods, and the diversity of these herbivorous arthropods increases, followed by increased predators. Please see Section $\mathbf{1 . 1}$ for the methods for testing this hypothesis.

性状独立互 在多基因型系统, 如果某些基因型的生态系统功能比 补效应它们在单基因型系统提高了, 但与基因型的功能性状 Trait-indepen- 无关，而且不以抑制其他基因型的适合度为代价(不同 dent comple- 基因型占据不重叠的生态位形成生态位互补或者基因 mentarity 型间存在正作用), 则为正效应。验证方法请见1.2节。 In genotypic mixture, if the ecosystem functions of some genotypes are improved than that in the genotypic monoculture but are not related to the functional traits of the genotypes, and are also not at the expense of inhibiting the fitness of other genotypes (different genotypes occupy non-overlapping niches to form niche complementarities or have positive effects between genotypes). It is a positive effect. Please see section $\mathbf{1 . 2}$ for the methods for testing this hypothesis.

性状依赖互 在多基因型系统, 如果具有特殊功能性状基因型(比 补效应如, 特殊功能性状使得基因型间形成嵌套生态位)的生 Trait-depen- 态功能比其在单基因型系统增加了, 且不以抑制其他 dent comple- 基因型适合度为代价, 则为正效应。验证方法请见 mentarity 1.2 节。

In genotypic mixture, if the ecological functions of genotypes with special functional traits (for example, the nested niches formed between genotypes) are increased than those of genotypic monoculture, and not at the expense of inhibiting the fitness of other genotypes, the effect is positive. Please see section $\mathbf{1 . 2}$ the methods for testing this hypothesis.

这些遗传多样性-生态系统功能关系(GD-EF)假说均从物种多样性-生态系统功能假说的短期效应假说迁移而来, 以解释遗传多样性对生态系统功能的 正效应，也是目前实验验证比较多的GD-EF机制假说。

All of these hypotheses of genetic diversity-ecological functioning (GD-EF) come from hypotheses of the short-term species diversity effects on ecosystem functioning to explain the positive effects of genetic diversity on ecosystem functioning, which are also the hypotheses of GD-EF that have been tested by many experiments.

(比如: 具有高浓度次生代谢物的基因型, 或者养分 低的基因型)的概率高于单基因型系统, 因此多基
因型系统的结构和功能优于单基因型系统, 这一效 应类似于从统计学角度分类的抽样效应(sampling

www.plant-ecology.com 
effect)(Tilman et al., 1997)。但优势效应和选择效应 也不等同, 其区别请见1.2节。非加性效应则是多基 因型系统中不同基因型间相互作用可能带来超出单 基因型系统的效应，也称为互补效应或交互效应， 包括生态位互补、基因型间正作用机制等(Loreau \& Hector, 2001; Fox, 2005)。对于遗传多样性对关联群 落节肢动物物种多样性的非加性效应, 有两种假说 来进一步解释。一是资源化假说: 大多数植食性节 肢动物表现出一定程度的食性偏好(Bernays \& Graham, 1988), 随着基因型多样性即食物多样性的 增加, 其相关联的植食性节肢动物多样性也增加 (Srivastava \& Lawton, 1998)。另一个是更多个体假 说: 如果地上净生产力随着基因型多样性升高, 那 么能够提供更多的能量给更多植食性节肢动物, 这 些植食性节肢动物多样性随之增加, 继而捕食者也 会增加(Srivastava \& Lawton, 1998)。

以月见草(Oenothera biennis)为研究对象的GD$\mathrm{EF}$ 实验结果表明, 基因型丰富度高(8-基因型)的样 方地上活植物组织的节肢动物多样性比单基因型样 方的高18\%, 基因型间的非加性效应和加性效应共 同起作用(Johnson et al., 2006)。以加拿大一枝黄花 (Solidago altissima) 为研究对象的GD-EF实验也有 类似的结论。基因型丰富度高的样方(12-基因型)比 单基因型样方的地上活植物组织的节肢动物多样性 高27\%, 但是只有非加性效应在起作用, 资源化假 说和更多个体假说共同解释非加性效应(Crutsinger et al., 2006)。而且非加性效应机制是基因型多样性 正效应的主要机制, 并不随季节变化(Crutsinger et al., 2008a)。但是, 基因型多样性对叶片枯落物的 节肢动物群落多样性无影响 (Crutsinger et al., 2008b)。因此, 遗传多样性对不同类型(叶片枯落物 和地上植物活组织)的节肢动物群落的生态效应可 能不一样。

目前, 检验基因型多样性对高级营养级生物群 落结构影响的加性效应和非加性效应方法如下：多 基因型组成系统中每个基因型植株的节肢动物多样 性期望值可以通过模拟方法得出, 即将随机抽取观 测到的单基因型样方中植株上的节肢动物种类, 组 合成多基因型组成系统的节肢动物多样性期望值。 如果实际多基因型组成系统中观测到的节肢动物多 样性平均值在所模拟系统节肢动物多样性的 $95 \%$ 置 信区间之外, 则是非加性效应起作用。同理, 对于节
肢动物的多度, 首先计算单基因型系统中每个植株 的节肢动物平均多度; 然后模拟系统的多度, 将每 个植株在单基因型系统的节肢动物的平均多度赋予 多基因型组成系统中对应基因型的植株; 再对比观 测到的节肢动物多度和模拟系统的节肢动物多度。 如果差异显著, 则是节肢动物的多度偏离加性效应, 而非加性效应在起作用(Johnson et al., 2006)。也可 以基于单基因型系统运用Monte Carlo模拟方法去 构建随机多基因型组成系统和它们的关联节肢动物 群落, 然后对比实测样方和随机多基因型组成系统 的关联节肢动物群落, 如果没有显著差异, 那么则 认为是加性效应机制在起作用, 反之则是非加性效 应机制(Crutsinger et al., 2006; Crawford \& Whitney, 2010)。这两种方法的逻辑原理一致, 两者均可用。 对于非加性效应中的更多个体假说的验证, 先采用 稀疏性方法(rarefaction)来校正每个系统的节肢动物 物种丰富度(因为每个系统采集到的节肢动物个体 数不一样), 如果被校正后的节肢动物物种丰富度 与系统生产力无关, 那么表明植物生产力通过影响 群落节肢动物个体数来控制节肢动物物种丰富度, 则支持更多个体假说; 资源化假说的验证则分析比 较相同基因型组成系统间以及不同基因型组成系统 间节肢动物物种组成相似度, 如果相同基因型组成 系统间物种组成相似，而不同基因型组成系统间物 种组成显著不同, 那么支持资源化假说(Crutsinger et al., 2006)。

\section{2 遗传多样性对生态系统功能: 初级生产力、养 分循环、稳定性的影响及机制}

与遗传多样性对生态系统结构的效应和机制类 似, 植物基因型多样性对于生态系统初级生产力、 养分循环、外来种入侵能力和生态系统稳定性也具 有正效应，其内在机制可能是非加性效应或者两种 效应均有(Reusch et al., 2005; Schweitzer et al., 2005; Crutsinger et al., 2006; Tomimatsu et al., 2014; Gerstenmaier et al., 2016; Sjoqvist \& Kremp, 2016), 单 独的加性效应较少。对于生态系统初级生产力, 北 美一枝黄花基因型丰富度最高的样方(12-基因型)比 单基因型样方的地上净初级生产力高36\%, 这是因 为非加性效应中的生态位互补机制在起作用 (Crutsinger et al., 2006)。同样, Reusch等(2005)研究 表明大叶藻(Zostera marina)的6-基因型样方比单基 因型样方的生物量提高了 $26 \%$, 其内在机制则是生 
态位互补和基因型间的正作用强于负抽样效应。另 外, 遗传多样性不仅对植物成年阶段, 而且对整个 生活史阶段(幼苗到成年)的表现都可能产生促进作 用。比如: 大叶藻基因型丰富度高的样方下一代幼 苗(种子繁殖)的地上和地下生物量比单基因型样方 更大，互补效应是主要机制(Hughes et al., 2016); 基 因型丰富度对拟南芥(Arabidopsis thaliana)的幼苗 萌发、生物量、花期和繁殖成功也有正效应, 是因 为加性效应和非加性效应共同起作用(Crawford \& Whitney, 2010)。

为了探讨物种多样性对生态系统功能的影响机 制, Loreau和Hector (2001)将多样性效应机制分离为 选择效应和互补效应, 通过比较选择效应和互补效 应与 0 的差异来判断效应的效果。Fox (2005)认为上 述方法中的选择效应包括了优势效应和性状依赖互 补效应，应该将其分开，否则高估选择效应的作用。 多样性效应被分为三部分(表2): 优势效应、性状依 赖互补效应和性状独立互补效应。近年较少研究运 用Fox (2005)的方法将遗传多样性效应细分为这三 部分(Hughes \& Stachowicz, 2011; Gerstenmaier et al., 2016; Hughes et al., 2016; Yang et al., 2017)。因此, 在今后GD-EF的机制研究时, 应多采用Fox (2005) 的方法。但由于肉眼难以区分不同基因型的植株个 体, 在实验后期需要借助分子标记来区分系统中不 同基因型的植株个体，工作量往往非常大。

对于养分循环, 研究表明遗传多样性同时促进 调落物分解(其中包括微生物和动物分解过程)和养 分再吸收。多基因型的落叶混合物具有更高的分解 速率而加速氮磷的释放 (Schweitzer et al., 2005; Wang et al., 2014), 而调落物养分含量影响分解速 率, 发草(Deschampsia cespitosa)基因型丰富度高的 样方的个体所产生的根系调落物氮含量比丰富度低 的样方高, 所以分解速率快, 幼苗吸收速率也高 (Semchenko et al., 2017)。中型动物也是植物碎屑的 主要分解者, 动物通过取食植物碎屑将其分解吸收, 是养分循环的重要过程之一。植物碎屑遗传多样性 协同食碎屑者物种多样性影响养分循环, 当植物碎 屑遗传多样性和消费者多样性均高时, 生物多样性 效应最高(Reynolds et al., 2017)。在单一动物物种群 落, 大叶藻碎屑的基因型丰富度不影响中型动物碎 屑取食; 而在动物物种多样性高的群落, 碎屑基因 型丰富度越高, 碎屑取食量越大, 分解速度越快,
而且中型动物存活率越高; 该研究结果归因于碎屑 动物对不同基因型碎屑的生态位分化(偏好)。

对于入侵物种的GD-EF研究不多。有研究发现 中国入侵种互花米草(Spartina alterniflora)基因型丰 富度提高了其最远扩散距离、斑块面积、斑块植株 数和生物量等扩散能力, 也抑制了本地种海三棱蔍 草(Scirpus mariqueter)的生长; 其内在机制是生态 位互补和抽样效应(Wang et al., 2012)。

随着全球变化形势愈来愈严重, 学者们越来越 关注植物GD-EF对环境变化的响应。在面对环境变 化时, 植物基因型丰富度往往能够提高生态系统的 稳定性(Hughes \& Stachowicz, 2004; Ehlers et al., 2008; Massa et al., 2013; McArt \& Thaler, 2013)。面 对干扰, 大叶藻基因型丰富度高的样方表现出更快 的恢复能力(Hughes \& Stachowicz, 2004); 面对全球 变暖, 大叶藻基因型丰富度能够抵抗全球变暖对生 态系统功能造成的负面影响(Ehlers et al., 2008); 面 对外来入侵种, 本地种植物基因型丰富度的增加能 减少和抵御外来昆虫啃食(McArt \& Thaler, 2013)。 但是, 遗传多样性维持生态系统功能稳定性的机制 不明确, 需要更深入的研究。

\section{3 功能多样性对遗传多样性与生态系统功能关 系的影响}

遗传多样性对生态系统功能的影响可能取决于 遗传多样性与表型性状变异(功能多样性)的关联程 度(Hughes et al., 2008), 基因型多样性的增加可能 会提高功能多样性(杨雪等, 2017), 从而加剧基因型 间对资源或空间利用的分化或者导致植食性动物产 生生态位分化, 因此影响GD-EF, 是互补效应产生 的原因之一。另一方面, 也有研究发现单基因型系 统, 功能多样性越高, 生态系统功能越高(Hughes, 2014), 那么从这个角度来说, 单基因型系统植株的 功能多样性也是加性效应产生的原因之一。大叶藻 基因型在功能性状(生长速率、营养吸收、光合效率、 多酚含量、对植食作用的敏感性和碎屑产量)表现出 不同, 造成植食性动物生态位分化, 可能是基因型 多样性正效应的机制(Hughes \& Stachowicz, 2004, 2011; Reusch et al., 2005; Massa et al., 2013)。但是如 果植物基因型在一个功能性状表现出来的差异还未 造成生态位分化, 那么功能多样性不一定影响到 GD-EF, 这样则需要测定多个功能性状来解释 GD-EF (Fischer et al., 2017; Jormalainen et al., 
2017)。而且基因型多样性和功能多样性对生态系统 功能的影响不是简单的相加关系, 而是具有交互作 用。有研究表明, 在功能多样性低时, 基因型多样性 正效应显著, 而在功能多样性高时, 基因型多样性 效应不明显(Hughes, 2014)。

因此, 在探究GD-EF内在机制时, 除了分开加 性效应和非加性效应, 还需深入探讨这些效应产生 的原因。因此需要测定多个功能性状特征, 从功能 多样性的角度进一步探讨遗传多样性效应的内在 机制。

\section{2 遗传多样性效应和物种多样性效应的比 较研究}

了解两个层次的生物多样性一一遗传多样性和 物种多样性及其交互作用对生态系统功能的相对重 要性, 能够更深入理解生物多样性效应。因为种内 性状变异往往小于种间变异, 所以一般认为遗传多 样性效应比物种多样性效应低。然而有研究表明基 因型多样性和物种多样性对生态系统功能的影响相 似。Cook-Patton等(2011)研究发现月见草基因型多 样性(8个基因型)和物种多样性(8个物种)均增加了 约 $17 \%$ 的初级生产力, 而且其机制均为生态位互补 效应; 基因型多样性和物种多样性也增加了节肢动 物群落物种多样性, 但其内在机制不一样, 基因型 多样性效应是通过更多个体假说来解释, 物种多样 性效应则是通过资源偏好假说来解释。因此, 我们 可能低估了自然生态系统中的遗传多样性效应。

在自然生态系统中, 物种多样性和遗传多样性 交织在一起, 所以基因型多样性和物种多样性的交 互效应非常重要。对于植物物种多样性高的生态系 统, Fridley和Grime (2010)同时操控基因型多样性和 物种多样性水平, 研究发现优势种基因型多样性高 时, 能够减轻物种间竞争, 从而有利于维持物种多 样性, 使得生态系统稳定, 但是对于生态系统初级 生产力却没有作用。而在植物物种多样性低的淡水 沙丘生态系统, 单独控制优势种基因型多样性或物 种多样性, 以及同时控制两种多样性及其交互作用 的实验结果表明: (1)在独立的基因型多样性实验中, 优势种植物Ammophila breviligulata基因型多样性对 节肢动物群落影响不明显。但在同时控制优势种基 因型多样性和物种多样性的实验中, 优势种基因型 多样性对节肢动物群落多样性的影响比物种多样性
要大, 其内在机制是非加性效应; 因此, 植物优势 种遗传多样性与物种多样性之间的交互作用, 能够 支持更丰富的关联节肢动物群落(Crawford \& Rudgers, 2013)。另外, 中国森林生物多样性与生态 系统功能研究项目(the BEF-China Project)中的二期 实验正在开展, 实验也涉及评估物种多样性和遗传 多样性相互关系对生态系统功能的影响(Bruelheide et al., 2014), 该研究将会加深对遗传多样性和物种 多样性交互效应的理解。(2)无论是基因型多样性还 是物种多样性, 两者单独对植物样方地上生物量并 没有显著影响, 但是其交互效应却改变了植物地上 部分生物量。物种多样性相同的样方, 随着优势种 基因型多样性的增加, 物种多样性和地上部分生物 量的关系由负相关转换成正相关 (Crawford \& Rudgers, 2012)。因此, 在自然生态系统尤其是物种 多样性较低的生态系统, 优势种遗传多样性与物种 多样性有可能共同维持更高水平的生态系统功能。

\section{3 遗传多样性-生态系统功能关系在生态保 护和修复中的运用}

遗传多样性越高抵御自然和人为的干扰, 恢 复、维持及促进生态系统功能的能力可能越强 (Hughes \& Stachowicz, 2004; Jahnke et al., 2015a; 申俊芳等, 2015)。因此, GD-EF也许能应用在生物多 样性保护、入侵种控制、应对全球变化和生态修复 实践中(Grady et al., 2011; Forsman, 2014; Kettenring et al., 2014)。对于保护已经局域灭绝或者种群正在 快速减少的物种, 往往需要重新引种, 在引种过程 中需要考虑到奠基者遗传多样性和引种地环境。奠 基者多样性对于种群的建立起到正效应，但遗传多 样性效应在自然环境比半自然或者标准实验室条件 下更强, 因为遗传多样性效应所需要的进化和生态 过程在复杂的自然环境中得到实现 (Forsman, 2014)。另一方面, 种群存在一定的局域适应性(local adaptation), 即存在适应局域生境的基因。因此, 引 种地环境与被引入种群自然生境相似可能会取得更 好的效果。

在应对物种入侵方面, 降低入侵种种群遗传多 样性, 提高本地种种群遗传多样性或许是一种控制 入侵种扩张的方法。有研究证明, 入侵种遗传多样 性越高则其入侵能力越强(Wang et al., 2012), 但本 地种遗传多样性高的样方能够更有效地抑制物种入 
侵。Yang等(2017)研究发现本地种Pseudoroegneria spicata遗传多样性高的样方中, 外来种Centaurea stoebe初级生产力减少 $44 \%$ 。在应对富营养化环境问 题方面, 有研究报道基因型多样性高的植物个体组 合能够有效降低营养盐浓度(Tomimatsu et al., 2014)。在应对气候变暖方面, 生态修复时运用本地 物种基因型以及来自温暖(或热带)地区的基因型以 提高生态系统初级生产力, 来适应未来快速气候变 暖的环境变化(Grady et al., 2011)。

在生态修复时, 小幅度地提高修复样方大叶藻 的基因多样性(此实验中用的是中性遗传多样性)能 够加快生态系统修复的速度、改善无脊椎动物生境 和提升生态系统服务功能(Reynolds et al., 2012)。但 是在生态修复中运用GD-EF时, 需要更长时间(3-5 年)、更大空间尺度 $\left(1000 \mathrm{~m}^{2}\right)$ 的多物种和多基因型交 互作用生态修复实验来支持 (Kettenring et al., 2014)。将GD-EF运用到生态修复时, 首先需要了解 所选择物种的交配系统一自交和异交以防止近交 或者远交衰退(McKay et al., 2005); 所选择物种是 否为多倍体, 因为植物同一物种的多倍体个体和二 倍体个体之间很可能存在生殖隔离(Husband \& Sabara, 2004), 如果存在生殖隔离则可能会导致下一 代遗传多样性降低因而影响生态系统功能。其次, 根据实际恢复目标来选择不同来源(品种、局域适应 性和遗传多样性)的种苗进行生态修复(Kettenring et al., 2014)。这3个来源各有优势, 品种来源能够快速 建立种群, 本地适应性种群来源能够保存本地适应 性基因, 遗传多样性种苗来源则能有效地恢复生态 系统功能。Kettenring等(2014)建议采用多个栽培品 种(提高遗传多样性)作为种苗来源或者将栽培品种 加入到遗传多样性种苗源中的方法, 特别适合需要 快速恢复植被和实现恢复生态系统功能的长期目标; 而结合局域适应性种群和遗传多样性种苗来源方法 最适合长期保存局域种群和恢复生态系统功能。然 而, 目前GD-EF的研究还有不足, 运用于生态保护 和修复中还需要实践来检验。

\section{4 研究不足与展望}

植物GD-EF研究虽然取得了不少进展, 主要表 现在对GD-EF关系格局和内在机制的探讨, 物种多 样性效应和遗传多样性效应大小比较及其交互作用 的研究, 以及GD-EF关系在生态修复中应用的初
探。但也存在不足之处, 其中以下 5 个方面更为突出。

\subsection{GD-EF研究需要进一步探讨其内在机制}

目前, GD-EF有关假说均从物种多样性-生态系 统功能关系迁移过来, 然而物种多样性和遗传多样 性水平上可能对生态系统功能影响不一样, 比如: (1)两个物种间基因基本无交流，而同一种群的不同 基因型之间则存在基因交流，如果出现近交衰退或 者远交衰退的现象(McKay et al., 2005), 即降低某 些有利于植物植株生长的基因在种群中的频率, 那 么可能会对生态系统结构功能产生负面影响。(2)在 种群水平上, 种群个体间可能存在亲缘选择(kin selection)作用(Jahnke et al., 2015b), 即亲缘关系越 近的个体越倾向于减少竞争作用, 而促进作用可能 增加, 进而可能提高生态系统功能。而在物种水平 上，由于亲缘关系越近的物种所需要的资源越相似， 那么亲缘关系越近的物种间竞争关系可能会更加激 烈, 从而可能降低生态系统功能(Cadotte et al., 2017)。但是物种多样性-生态系统功能关系迁移过 来的假说, 还未考虑到物种多样性效应和遗传多样 性效应这两方面的差别, 可能需要将种群交配系 统、亲缘度、染色体倍性等因素考虑到相关假说中 以修正相关假说, 并开展多世代长期实验, 以探讨 是否有别于物种多样性-生态系统功能关系的机制。 另外, 物种多样性-生态系统功能关系机制中的长 期效应假说一统计均衡效应、保险假说和圥余机 制(张全国和张大勇, 2002)是否也能用来解释GD-EF 的长期效应有待验证。

\section{2 未评估遗传多样性对生态系统多功能性的影响}

物种多样性与生态系统功能关系的研究已经进 展到评估物种多样性和系统发育多样性对生态系统 多功能性的影响(Jing et al., 2015; Lefcheck et al., 2015; 徐炜等, 2016b; Cadotte et al., 2017; Gamfeldt \& Roger, 2017)。生态系统多功能性是指生态系统同 时维持多种生态系统功能和服务的能力, 生态系统 功能之间的权衡可能会导致生态系统同时提供多个 功能的能力降低(Hector \& Bagchi, 2007; 徐炜等, 2016b), 那么一个生态系统需要多少物种多样性才 能同时维持多个生态系统功能? 而对于单一优势植 物种形成的生态系统, 我们更关心的是需要多少基 因型多样性才能同时维持多个生态系统功能? 目前, GD-EF的研究还停留在基因型多样性对单个生态系 统功能的影响, 并未涉及同时观测多种生态系统功 
能、测度基因型多样性对生态系统多功能性的影响 以及探讨生态系统多功能性的维持机制。近来, 学 者们发展出了多种生态系统多功能性指数, 比如: 单功能法(Duffy et al., 2003)、功能-物种替代法 (Hector \& Bagchi, 2007)、平均值法(Duffy et al., 2003)、单阈值法(Gamfeldt et al., 2008)、多阈值法 (Byrnes et al., 2014)和多元模型法(Dooley et al., 2015)。其中, 多阈值法和多元模型法为最新、具有 明显优点及互补的测度方法。多阈值法评估的是多 样性的增加达到某一阈值水平时功能数的变化, 其 克服单阈值法很难准确地选择恰当阈值的缺点, 并 能够得出整体功能何时达到最大值; 多元模型法则 考虑了不同方面的多样性(丰富度、均匀度及组成等) 对多种生态系统功能的影响, 比较多样性和环境因 素对生态系统功能的相对重要性, 而且还可以分析 功能之间的权衡和协同等关系(Byrnes et al., 2014; Dooley et al., 2015; 徐炜等, 2016a)。这些方法目前 已经应用于评估物种多样性对生态系统多功能性的 影响, 未来研究工作可以采用和改进这些研究方法 来尝试评估遗传多样性对生态系统多功能性的影响。

\section{3 较少探讨不同遗传多样性度量对生态系统功 能的影响}

大多数GD-EF实验均采用基因型丰富度作为遗 传多样性的测度指标, 即在实验操作中选择不同数 目的克隆基因型作为基因型丰富度。在实验设计和 分析中较少探讨基因丰富度、基因型均匀度、基因 型相异度或者基因型亲缘度对生态系统功能的影响, 而基因型丰富度可能不能完全代表遗传变异, 基因 型均匀度、基因丰富度、组成、相异度和亲缘度等 也是遗传多样性的不同方面(Hughes et al., 2008; Jousset et al., 2011)。

有些研究在一定程度上探讨不同遗传多样性度 量对生态系统功能的影响。比如: Abbott等(2017)研 究表明基因型丰富度对于大叶藻生物量累积的作用 最强; 基因型多样性(丰富度+均匀度)比单独基因 型丰富度对生物量的影响更大, 但是基因型亲缘度 独立于基因型多样性对大叶藻生物量具有负作用。

然而Stachowicz等(2013)发现基因型亲缘关系对大 叶藻生物量累积具正效应。Hughes等(2016)也发现 大叶藻幼苗基因型亲缘度独立于遗传多样性与幼苗 形态特征存在单峰格局, 在基因型亲缘度中等时, 幼苗具有多的叶片和更长的根。因此, 基因型亲缘
度对生态系统功能关系的影响未明确, 需要进一步 研究。

Massa等(2013)对比海草(Zostera noltii)的基因 丰富度和基因型丰富度对生态稳定性和恢复力的影 响时发现，基因丰富度和基因型丰富度对海草床的 稳定性和恢复力均具有正向作用, 而且基因丰富度 的作用更强。因此, 在实验设计时应该考虑到基因 型丰富度和基因丰富度之间的关系, 避免暗处理, 否则可能会存在基因丰富度低但基因型丰富度高的 实验处理, 那么基因型丰富度的效应往往被低估。 再者, Whitlock (2014)通过整合分析发现适应性遗 传多样性与生态系统结构和功能的关系是正相关关 系, 而中性遗传多样性则与生态系统结构和功能无 关。但是, Whitlock (2014) 将克隆多样性、生态型多 样性、品种数目等看作适应性遗传多样性, 这样的 分类和定义不精确。另外, Whitlock (2014)分析中性 遗传多样性与生态系统结构关系所用到的数据大部 分是关于遗传多样性与群落中植物物种多样性(同 一营养级生态系统结构) 关系研究的, 而缺乏中性 遗传多样性与高营养级生物群落物种多样性关系的 数据, 所以Whitlock (2014)的结论也有待商榷。因此, 未来实验研究中, 应该更多地开展不同遗传多样性 测度对生态系统结构和功能影响的研究, 并比较它 们对生态系统功能影响的大小, 以便更全面地理解 GD-EF关系格局及机制。

\section{4 缺少长期的和多空间尺度结合的遗传多样性 和生态系统功能关系实验}

对生态学研究来说, 长期实验是验证和整合各 类观点的重要手段。物种多样性和生态系统功能实 验通过长期实验达成共识: 物种多样性对生态系统 功能具有正向作用, 而抽样效应和生态位互补效应 均是影响物种多样性和生态系统功能关系的重要机 制, 只是在不同的时间尺度, 不一样的机制在起决 定作用; 即在实验的前期抽样效应起决定作用, 而 随着时间的推移, 物种生态位补偿效应逐渐凸显其 重要性(Cardinale et al., 2007)。目前GD-EF实验时间 尺度非常短(1-2年), 然而进化的时间尺度非常长, 对于GD-EF相关理论的验证更需要开展长期实验, 随着实验时间尺度的拉长, 可能得到不一样的结 果。长远(多个世代)来说, 如果未发生近交或者远交 衰退, 遗传多样性效应随着时间的推移而增强, 非 加性效应越来越重要。但是如果发生近交或者远交 
衰退，基因通过杂交稀释局域适应性基因型频率， 那么遗传多样性效应可能会随时间的推移而减弱。 另一方面, 在当前的GD-EF实验中, 选择的基因型 往往是来自地理距离非常远的种群或者特意选择表 型差异非常大的种群, 而实验却在非常小的空间尺 度开展, 这样的实验不能反映自然条件下遗传多样 性对生态系统功能的作用, 也忽略了环境因素和空 间因素对生态系统功能的影响(Tack et al., 2010, 2012)。因此, 植物基因型和环境变异的空间尺度应 该对应起来, 在选择基因型和实验地点时随机取样, 并采用多尺度结合的方法, 即在局域和区域尺度的 不同地点进行重复实验, 然后采用混合模型将基因 型多样性、环境因素、空间因素及其交互作用的作 用大小分离出来。

\section{5 较少比较遗传多样性的生态效应相对于其他 因子的作用大小}

虽然大多数GD-EF控制实验证明遗传多样性对 高营养级生物群落结构和生态系统功能为正向作用, 但是有关这种作用在自然界中相对于其他因子的重 要性的研究比较少, 这类研究可以通过操控多种因 子来实现(Hughes et al., 2008; Hersch-Green et al., 2011)。比如: 通过同时操控土壤养分和遗传多样性 水平的实验, Crutsinger等(2013)研究发现土壤养分 因子比种内(Baccharis pilularis)遗传变异对林下植 物群落多样性、盖度和生物量的影响更为重要; Souza等(2017)则发现北美一枝黄花基因型多样性 比养分对初级生产力、碳循环和水分交换产生的影 响更大。目前这些研究结果不一致, 还需要开展更 多的多因子控制实验以取得普遍性的结论。

此外, 建议在物种多样性-生态系统功能实验 平台或者生态系统对全球气候变化(如增温、降雨格 局、氮沉降等)响应等野外控制实验平台中加入优势 种遗传多样性的监测, 也将遗传多样性指标纳入相 关数据分析中; 同时建立自然条件下GD-EF野外长 期监测平台。而将遗传多样性对生态系统功能的正 效应运用在生态修复中可能还需要多尺度结合的研 究以及生态修复实践来完善修复方法。

\section{参考文献}

Abbott JM, Grosberg RK, Williams SL, Stachowicz JJ (2017). Multiple dimensions of intraspecific diversity affect biomass of eelgrass and its associated community. Ecology, 98,
3152-3164.

Bailey JK (2011). From genes to ecosystems: A genetic basis to ecosystem services. Population Ecology, 53, 47-52.

Bailey JK, Schweitzer JA, Ubeda F, Koricheva J, LeRoy CJ, Madritch MD, Rehill BJ, Bangert RK, Fischer DG, Allan GJ, Whitham TG (2009). From genes to ecosystems: A synthesis of the effects of plant genetic factors across levels of organization. Philosophical Transactions of the Royal Society B-Biological Sciences, 364, 1607-1616.

Bernays E, Graham M (1988). On the evolution of host specificity in phytophagous arthropods. Ecology, 69, 886-892.

Bruelheide H, Nadrowski K, Assmann T, Bauhus J, Both S, Buscot F, Chen XY, Ding BY, Durka W, Erfmeier A, Gutknecht JLM, Guo DL, Guo LD, Hardtle W, He JS, Klein AM, Kuhn P, Liang Y, Liu XJ, Michalski S, Niklaus PA, Pei KQ, Scherer-Lorenzen M, Scholten T, Schuldt A, Seidler G, Trogisch S, von Oheimb G, Welk E, Wirth C, Wubet T, Yang XF, Yu MJ, Zhang SR, Zhou HZ, Fischer M, Ma KP, Schmid B (2014). Designing forest biodiversity experiments: General considerations illustrated by a new large experiment in subtropical China. Methods in Ecology and Evolution, 5, 74-89.

Butchart SHM, Walpole M, Collen B, van Strien A, Scharlemann JPW, Almond REA, Baillie JEM, Bomhard B, Brown C, Bruno J, Carpenter KE, Carr GM, Chanson J, Chenery AM, Csirke J, Davidson NC, Dentener F, Foster M, Galli A, Galloway JN, Genovesi P, Gregory RD, Hockings M, Kapos V, Lamarque JF, Leverington F, Loh J, McGeoch MA, McRae L, Minasyan A, Morcillo MH, Oldfield TEE, Pauly D, Quader S, Revenga C, Sauer JR, Skolnik B, Spear D, Stanwell-Smith D, Stuart SN, Symes A, Tierney M, Tyrrell TD, Vie JC, Watson R (2010). Global biodiversity: Indicators of recent declines. Science, 328, 1164-1168.

Byrnes JEK, Gamfeldt L, Isbell F, Lefcheck JS, Griffin JN, Hector A, Cardinale BJ, Hooper DU, Dee LE, Duffy JE (2014). Investigating the relationship between biodiversity and ecosystem multifunctionality: Challenges and solutions. Methods in Ecology and Evolution, 5, 111-124.

Cadotte MW, Livingstone SW, Yasui SLE, Dinnage R, Li JT, Marushia R, Santangelo J, Shu W (2017). Explaining ecosystem multifunction with evolutionary models. Ecology, 98, 3175-3187.

Cardinale BJ, Wright JP, Cadotte MW, Carroll IT, Hector A, Srivastava DS, Loreau M, Weis JJ (2007). Impacts of plant diversity on biomass production increase through time because of species complementarity. Proceedings of the $\mathrm{Na}$ tional Academy of Sciences of the United States of America, 104, 18123-18128.

Cook-Patton SC, McArt SH, Parachnowitsch AL, Thaler JS, Agrawal AA (2011). A direct comparison of the consequences of plant genotypic and species diversity on

www.plant-ecology.com 
communities and ecosystem function. Ecology, 92, 915-923.

Crawford KM, Rudgers JA (2012). Plant species diversity and genetic diversity within a dominant species interactively affect plant community biomass. Journal of Ecology, 100, 1512-1521.

Crawford KM, Rudgers JA (2013). Genetic diversity within a dominant plant outweighs plant species diversity in structuring an arthropod community. Ecology, 94, 1025-1035.

Crawford KM, Whitney KD (2010). Population genetic diversity influences colonization success. Molecular Ecology, 19, 1253-1263.

Crutsinger GM, Carter BE, Rudgers JA (2013). Soil nutrients trump intraspecific effects on understory plant communities. Oecologia, 173, 1531-1538.

Crutsinger GM, Collins MD, Fordyce JA, Gompert Z, Nice CC, Sanders NJ (2006). Plant genotypic diversity predicts community structure and governs an ecosystem process. Science, 313, 966-968.

Crutsinger GM, Collins MD, Fordyce JA, Sanders NJ (2008a). Temporal dynamics in non-additive responses of arthropods to host-plant genotypic diversity. Oikos, 117, 255-264.

Crutsinger GM, Reynolds WN, Classen AT, Sanders NJ (2008b). Disparate effects of plant genotypic diversity on foliage and litter arthropod communities. Oecologia, 158, 65-75.

Darwin C (1859). On the Origin of Species by Means of Natural Selection. J. Murray, London.

Dooley A, Isbell F, Kirwan L, Connolly J, Finn JA, Brophy C (2015). Testing the effects of diversity on ecosystem multifunctionality using a multivariate model. Ecology Letters, 18, 1242-1251.

Duffy JE, Richardson JP, Canuel EA (2003). Grazer diversity effects on ecosystem functioning in seagrass beds. Ecology Letters, 6, 637-645.

Ehlers A, Worm B, Reusch TBH (2008). Importance of genetic diversity in eelgrass Zostera marina for its resilience to global warming. Marine Ecology Progress Series, 355, $1-7$.

Fischer DG, Wimp GM, Hersch-Green E, Bangert RK, Leroy CJ, Bailey JK, Schweitzer JA, Dirks C, Hart SC, Allan GJ, Whitham TG (2017). Tree genetics strongly affect forest productivity, but intraspecific diversity-productivity relationships do not. Functional Ecology, 31, 520-529.

Forsman A (2014). Effects of genotypic and phenotypic variation on establishment are important for conservation, invasion, and infection biology. Proceedings of the National Academy of Sciences of the United States of America, 111, 302-307.

Fox JW (2005). Interpreting the "selection effect" of biodiversity on ecosystem function. Ecology Letters, 8, 846-856.
Frasier TR (2008). STORM: Software for testing hypotheses of relatedness and mating patterns. Molecular Ecology Resources, 8, 1263-1266.

Fridley JD, Grime JP (2010). Community and ecosystem effects of intraspecific genetic diversity in grassland microcosms of varying species diversity. Ecology, 91, 2272-2283.

Gamfeldt L, Hillebrand H, Jonsson PR (2008). Multiple functions increase the importance of biodiversity for overall ecosystem functioning. Ecology, 89, 1223-1231.

Gamfeldt L, Roger F (2017). Revisiting the biodiversityecosystem multifunctionality relationship. Nature Ecology \& Evolution, 1, 168. DOI: 10.1038/s41559-017-0168.

Gerstenmaier CE, Krueger-Hadfield SA, Sotka EE (2016). Genotypic diversity in a non-native ecosystem engineer has variable impacts on productivity. Marine Ecology Progress Series, 556, 79-89.

Grady KC, Ferrier SM, Kolb TE, Hart SC, Allan GJ, Whitham TG (2011). Genetic variation in productivity of foundation riparian species at the edge of their distribution: Implications for restoration and assisted migration in a warming climate. Global Change Biology, 17, 3724-3735.

Hector A, Bagchi R (2007). Biodiversity and ecosystem multifunctionality. Nature, 448, 188-190.

Hersch-Green EI, Turley NE, Johnson MTJ (2011). Community genetics: What have we accomplished and where should we be going? Philosophical Transactions of the Royal Society B-Biological Sciences, 366, 1453-1460.

Huang K, Ritland K, Guo ST, Dunn DW, Chen D, Ren Y, Qi XG, Zhang P, He G, Li BG (2015). Estimating pairwise relatedness between individuals with different levels of ploidy. Molecular Ecology Resources, 15, 772-784.

Hughes AR (2014). Genotypic diversity and trait variance interact to affect marsh plant performance. Journal of Ecology, 102, 651-658.

Hughes AR, Hanley TC, Schenck FR, Hays CG (2016). Genetic diversity of seagrass seeds influences seedling morphology and biomass. Ecology, 97, 3538-3546.

Hughes AR, Inouye BD, Johnson MTJ, Underwood N, Vellend $M$ (2008). Ecological consequences of genetic diversity. Ecology Letters, 11, 609-623.

Hughes AR, Stachowicz JJ (2004). Genetic diversity enhances the resistance of a seagrass ecosystem to disturbance. Proceedings of the National Academy of Sciences of the United States of America, 101, 8998-9002.

Hughes AR, Stachowicz JJ (2011). Seagrass genotypic diversity increases disturbance response via complementarity and dominance. Journal of Ecology, 99, 445-453.

Husband BC, Sabara HA (2004). Reproductive isolation between autotetraploids and their diploid progenitors in fireweed, Chamerion angustifolium (Onagraceae). New Phytologist, 161, 703-713.

Huston MA (1997). Hidden treatments in ecological experiments: 
Re-evaluating the ecosystem function of biodiversity. Oecologia, 110, 449-460.

Jahnke M, Olsen JL, Procaccini G (2015a). A meta-analysis reveals a positive correlation between genetic diversity metrics and environmental status in the long-lived seagrass Posidonia oceanica. Molecular Ecology, 24, 2336-2348.

Jahnke M, Pages JF, Alcoverro T, Lavery PS, McMahon KM, Procaccini G (2015b). Should we sync? Seascape-level genetic and ecological factors determine seagrass flowering patterns. Journal of Ecology, 103, 1464-1474.

Jing X, Sanders NJ, Shi Y, Chu HY, Classen AT, Zhao K, Chen LT, Shi Y, Jiang YX, He JS (2015). The links between ecosystem multifunctionality and above- and belowground biodiversity are mediated by climate. Nature Communications, 6, 8159. DOI: 10.1038/ncomms9159.

Johnson MTJ, Lajeunesse MJ, Agrawal AA (2006). Additive and interactive effects of plant genotypic diversity on arthropod communities and plant fitness. Ecology Letters, 9, 24-34.

Jormalainen V, Danelli M, Gagnon K, Hillebrand H, Rothausler E, Salminen JP, Sjoroos J (2017). Genetic variation of a foundation rockweed species affects associated communities. Ecology, 98, 2940-2951.

Jousset A, Schmid B, Scheu S, Eisenhauer N (2011). Genotypic richness and dissimilarity opposingly affect ecosystem functioning. Ecology Letters, 14, 537-545.

Kettenring KM, Mercer KL, Adams CR, Hines J (2014). Application of genetic diversity-ecosystem function research to ecological restoration. Journal of Applied Ecology, 51, 339-348.

Lande R (1988). Genetics and demography in biological conservation. Science, 241, 1455-1460.

Lefcheck JS, Byrnes JEK, Isbell F, Gamfeldt L, Griffin JN, Eisenhauer N, Hensel MJS, Hector A, Cardinale BJ, Duffy JE (2015). Biodiversity enhances ecosystem multifunctionality across trophic levels and habitats. Nature Communications, 6, 6936. DOI: 10.1038/ncomms6936.

Loreau M, Hector A (2001). Partitioning selection and complementarity in biodiversity experiments. Nature, 413, 548-548.

Massa SI, Paulino CM, Serrao EA, Duarte CM, Arnaud-Haond $S$ (2013). Entangled effects of allelic and clonal (genotypic) richness in the resistance and resilience of experimental populations of the seagrass Zostera noltii to diatom invasion. BMC Ecology, 13, 1-12.

McArt SH, Thaler JS (2013). Plant genotypic diversity reduces the rate of consumer resource utilization. Proceedings of the Royal Society B-Biological Sciences, 280, 20130639. DOI: 10.1098/rspb.2013.0639

McKay JK, Christian CE, Harrison S, Rice KJ (2005). "How local is local?"-A review of practical and conceptual issues in the genetics of restoration. Restoration Ecology,
13, 432-440.

Pew J, Muir PH, Wang JL, Frasier TR (2015). Related: An R package for analysing pairwise relatedness from codominant molecular markers. Molecular Ecology Resources, 15, 557-561.

Reusch TBH, Ehlers A, Hammerli A, Worm B (2005). Ecosystem recovery after climatic extremes enhanced by genotypic diversity. Proceedings of the National Academy of Sciences of the United States of America, 102, 2826-2831.

Reynolds LK, Chan KM, Huynh E, Williams SL, Stachowicz JJ (2017). Plant genotype identity and diversity interact with mesograzer species diversity to influence detrital consumption in eelgrass meadows. Oikos, 127, 327-336.

Reynolds LK, McGlathery KJ, Waycott M (2012). Genetic diversity enhances restoration success by augmenting ecosystem services. PLOS ONE, 7, e38397. DOI: 10.1371/journal.pone.0038397.

Schweitzer JA, Bailey JK, Hart SC, Whitham TG (2005). Nonadditive effects of mixing cottonwood genotypes on litter decomposition and nutrient dynamics. Ecology, 86, 2834-2840.

Semchenko M, Saar S, Lepik A (2017). Intraspecific genetic diversity modulates plant-soil feedback and nutrient cycling. New Phytologist, 216, 90-98.

Shen DW, Li YY, Chen XY (2007). Review of clonal diversity and its effects on ecosystem functioning. Journal of Plant Ecology (Chinese Version), 31, 552-560. [沈栋伟, 李媛 媛, 陈小勇 (2007). 植物克隆多样性与生态系统功能. 植物生态学报, 31, 552-560.]

Shen JF, Ren HQ, Xin XJ, Xu B, Gao YB, Zhao NX (2015). Leymus chinensis genotypic diversity increases the response of populations to disturbance. Acta Ecologica Sinica, 35, 7682-7689. [申俊芳, 任慧琴, 辛晓静, 徐冰, 高玉葆, 赵念席 (2015). 羊草基因型多样性能增强种群 对干扰的响应. 生态学报, 35, 7682-7689.]

Shen JF, Xin XJ, Zhao NX, Gao YB (2016). Effects of genotypic diversity of Leymus chinensis population on soil macrofauna and microorganism community. Chinese Journal of Ecology, 35, 1226-1232. [申俊芳, 辛晓静, 赵 念席, 高玉荣 (2016). 羊草基因型数目对地下动物及微 生物群落的影响. 生态学杂志, 35, 1226-1232.]

Sjoqvist CO, Kremp A (2016). Genetic diversity affects ecological performance and stress response of marine diatom populations. The ISME Journal, 10, 2755-2766.

Smithson JB, Lenné JM (1996). Varietal mixtures: A viable strategy for sustainable productivity in subsistence agriculture. Annals of Applied Biology, 128, 127-158.

Souza L, Stuble KL, Genung MA, Classen AT (2017). Plant genotypic variation and intraspecific diversity trump soil nutrient availability to shape old-field structure and function. Functional Ecology, 31, 965-974.

Srivastava DS, Lawton JH (1998). Why more productive sites

www.plant-ecology.com 
have more species: An experimental test of theory using tree-hole communities. The American Naturalist, 152, 510-529.

Stachowicz JJ, Kamel SJ, Hughes AR, Grosberg RK (2013). Genetic relatedness influences plant biomass accumulation in eelgrass (Zostera marina). The American Naturalist, 181, 715-724.

Tack AJM, Johnson MTJ, Roslin T (2012). Sizing up community genetics: It's a matter of scale. Oikos, 121, 481-488.

Tack AJM, Ovaskainen O, Pulkkinen P, Roslin T (2010). Spatial location dominates over host plant genotype in structuring an herbivore community. Ecology, 91, 2660-2672.

Tilman D, Isbell F, Cowles JM (2014). Biodiversity and ecosystem functioning. Annual Review of Ecology, Evolution, and Systematics, 45, 471-493.

Tilman D, Lehman CL, Thomson KT (1997). Plant diversity and ecosystem productivity: Theoretical considerations. Proceedings of the National Academy of Sciences of the United States of America, 94, 1857-1861.

Tomimatsu H, Nakano K, Yamamoto N, Suyama Y (2014). Effects of genotypic diversity of Phragmites australis on primary productivity and water quality in an experimental wetland. Oecologia, 175, 163-172.

Wang XY, Miao Y, Yu S, Chen XY, Schmid B (2014). Genotypic diversity of an invasive plant species promotes litter decomposition and associated processes. Oecologia, 174, 993-1005.

Wang XY, Shen DW, Jiao J, Xu NN, Yu S, Zhou XF, Shi MM, Chen XY (2012). Genotypic diversity enhances invasive ability of Spartina alterniflora. Molecular Ecology, 21, 2542-2551.

Whitham TG, Bailey JK, Schweitzer JA, Shuster SM, Bangert RK, Leroy CJ, Lonsdorf EV, Allan GJ, DiFazio SP, Potts BM, Fischer DG, Gehring CA, Lindroth RL, Marks JC, Hart SC, Wimp GM, Wooley SC (2006). A framework for community and ecosystem genetics: From genes to ecosystems. Nature Reviews Genetics, 7, 510-523.

Whitlock R (2014). Relationships between adaptive and neutral genetic diversity and ecological structure and functioning: A meta-analysis. Journal of Ecology, 102, 857-872.

Wolfe MS (1985). The current status and prospects of multiline cultivars and variety mixtures for disease resistance. Annual Review of Phytopathology, 23, 251-273.

Xu W, Jing X, Ma ZY, He JS (2016a). A review on the measurement of ecosystem multifunctionality. Biodiversity Science, 24, 72-84. [徐炜, 井新, 马志远, 贺金生 (2016a). 生态系统多功能性的测度方法. 生物多样性, 24, 72-84.]

Xu W, Ma ZY, Jing X, He JS (2016b). Biodiversity and ecosystem multifunctionality: Advances and perspectives. Biodiversity Science, 24, 55-71. [徐炜, 马志远, 井新, 贺金生 (2016b). 生物多样性与生态系统多功能性:进 展与展望. 生物多样性, 24, 55-71.]

Yang LX, Callaway RM, Atwater DZ (2017). Ecotypic diversity of a dominant grassland species resists exotic invasion. Biological Invasions, 19, 1483-1493.

Yang X, Shen JF, Zhao NX, Gao YB (2017). Phenotypic plasticity and genetic differentiation of quantitative traits in genotypes of Leymus chinensis. Chinese Journal of Plant Ecology, 41，359-368. [杨雪, 申俊芳, 赵念席, 高玉葆 (2017). 不同基因型羊草数量性状的可塑性及遗传分化. 植物生态学报, 41, 359-368.]

Zhang QG, Zhang DY (2002). Biodiversity and ecosystem functioning: Recent advances and trends. Biodiversity Science, 11, 351-363. [张全国, 张大勇 (2003). 生物多 样性与生态系统功能: 进展与争论. 生物多样性, 11 , 351-363.] 